\title{
Validade Fatorial Confirmatória e Consistência Interna da Escala Global de Crenças no Mundo Justo - GJWS
}

\author{
Valdiney V. Gouveia \\ Universidade Federal da Paraíba \\ Carlos Eduardo Pimentel \\ Universidade de Brasília \\ Jorge Artur P. de M. Coelho \\ Universidade Federal de Alagoas \\ Viviane Andrade Prado Maynart \\ Tamara dos Santos Mendonça \\ Universidade Tiradentes
}

\begin{abstract}
RESUMO
A crença no mundo justo é um construto fundamental para a justiça desde uma perspectiva psicossocial. No entanto, poucos pesquisadores na psicologia brasileira têm se interessado no tema. Com o fim de contribuir com esta área, buscou-se conhecer a validade e precisão da Escala Global de Crenças no Mundo Justo (GJWS), verificando a importância do sexo, idade, curso, período, nível de religiosidade, estado civil e renda percebida para explicar sua variabilidade. Esta medida apresentou originalmente qualidades psicométricas satisfatórias, além de ser de fácil e rápida aplicação. Um total de 254 estudantes de uma universidade particular, da cidade de Aracaju (Sergipe), com média de idade de 21,8 anos $(\mathrm{dp}=6,34)$, sendo a maioria mulher, solteira, de classe média, dos cursos de Direito e Psicologia, respondeu a GJWS - uma medida breve e unifatorial. Esta se compõe de sete itens destinados a aferir crenças no mundo justo, sendo respondidos numa escala do tipo Likert, de 6 pontos, que variam de $1=$ Discordo totalmente a $6=$ Concordo totalmente. Os principais resultados mostram que a GJWS é uma medida válida e precisa, portanto, pode ser utilizada em estudos sobre o tema. Não se verificou importância de variáveis demográficas para explicar suas pontuações. Por fim, apontam-se as limitações do presente estudo, indicando-se direções de pesquisas futuras.
\end{abstract}

Palavras-chave: validade; precisão; crença no mundo justo; justiça.

\section{ABSTRACT \\ Confirmatory Factorial Validity and Reliability of the Global Belief in a Just World Scale - GJWS}

The belief in a just world is an essential construct for justice from a psychosocial perspective. However, only a few researchers in Brazilian psychology have being interested on this theme. In order to contribute to this subject, this study aimed at knowing the validity and reliability of the Global Belief in a Just World Scale (GJWS), and to verify the importance of gender, age, course, grade level, religiosity degree, marital status and income to account for its scores. This measure presented originally good psychometric properties, besides being of simple and rapid administration. Participants were 254 undergraduate students of a private university from Aracaju (Sergipe), with mean age of 21.8 years $(\mathrm{sd}=6.34)$, most of them female, single, middle-class, attending the courses of Law and Psychology. They filled the GJWS - a unifactor brief measure, composed by seven items that assess the beliefs in a just world, rated on a 6-point Likert scale, ranging from $1=$ Strongly disagree to $6=$ Strongly agree. The main results showed that the GJWS is a valid and reliable instrument, and can be used in studies on this theme. The demographic variables didn't show the expected importance though. Finally, the limitations of the current research and directions for future studies are discussed.

Keywords: validity; reliability; belief in a just world; justice. 
Grandes parcelas da humanidade vivenciam, hoje, um sentimento geral de injustiça social, econômica, cultural e até mesmo religiosa. Por ser um tema cada vez mais fundamental da vida social, o estudo da justiça passou a integrar as preocupações teóricas dos analistas sociais, especificamente dos psicólogos sociais (Rodrigues, Assmar \& Jablonski, 2000). Porém, os autores afirmam que este ainda é um tema pouco explorado no Brasil, sendo muito mais estudado em contexto norte-americano.

\section{As crenças no mundo justo}

Diariamente, os jornais noticiam todo tipo de tragédias, por exemplo, catástrofes naturais (enchentes, abalo sísmico) e crimes (assassinatos, sequestros). Uma maneira que os indivíduos utilizam para lidar com estes inquietantes lembretes de que coisas ruins acontecem, é explicá-las, fazer com que pareça que elas jamais poderiam acontecer a eles mesmos. Faz-se isso acreditando que coisas ruins acontecem apenas a pessoas más. Deve-se a Melvin J. Lerner (1980) a sistematização desta teoria, denominada de crença em um mundo justo (Belief in a Just World - BJW), que consiste na suposição de que o indivíduo recebe o que merece e merece o que recebe (Aronson, Wilson \& Akert, 2002; Montada, 2003).

Há muito se tem acumulado evidências para a hipótese da BJW (Furnhan, 2003; Furnham \& Procter, 1989; Lerner, 1980; Lerner \& Miller, 1978). As crenças no mundo justo vêm sendo relacionadas com alguns construtos, como atitudes frente à deficiência $\mathrm{e}$ à caridade (Furnham, 1995), a religiosidade, bem-estar subjetivo, atitudes políticas (Dalbert, Lipkus, Sallay \& Goch, 2001), confiança interpessoal e assistência religiosa (Bègue, 2002), traços de personalidade e valores (Wolfradt \& Dalbert, 2003), autoritarismo e ética de trabalho protestante (Mudrack, 2005), reações agressivas (Bègue \& Muller, 2006) e perdão (Strelan, 2007). Quanto às variáveis sociodemográficas, Furnhan (2003) revisou vários estudos que mostram relações das crenças no mundo justo com o sexo dos participantes, zona urbana e rural, trabalho, etnia, status socioeconômico, religião e religiosidade.

A teoria da crença no mundo justo teve na sua origem a constatação do fenômeno de "culpar a vítima" (Correia \& Vala, 2003; Lerner, 2003). Estudos experimentais demonstram que as vítimas de crimes e acidentes são muitas vezes consideradas responsáveis pelo que lhes aconteceu (Aronson \& cols., 2002; Montada, 2003). Este fenômeno tem sido identificado em diferentes situações de culpabilização da vítima (Edlund, Sagarin \& Johnson, 2007; Furnham, 2003; Lerner, 2003; Otto, Boos, Dalbert, Schöps \& Hoyer, 2006). Ao utilizar esta tendenciosidade de atribuição, o observador evita reconhecer que há certa aleatoriedade na vida e mantém distantes pensamentos preocupantes com a própria segurança (Aronson \& cols., 2002; Montada, 2003). De tal modo, considerar a vítima inocente é incongruente com a crença no mundo justo, pelo contrário, congruente é que a responsabilidade da vitimização resida na própria vítima, isto é, que a vítima seja não-inocente; "as coisas más acontecem a pessoas más" (Lerner, 1998, p. 251). Portanto, a crença no mundo justo é uma atribuição defensiva que ajuda as pessoas a se manterem confiantes na segurança de suas vidas. $O$ contexto também influencia esse processo de atribuição. De acordo com Furnham (2003), nos países subdesenvolvidos, em que prevalecem as desigualdades econômicas e sociais, as pessoas tendem a acreditar que os pobres e desprivilegiados têm menos porque merecem menos. As desigualdades são consideradas "legítimas". Desse modo, a atribuição de um mundo justo pode ser usada para explicar e justificar a injustiça (Aronson \& cols., 2002). Furnham destaca ademais que em contextos culturais, em que prevalecem extremos de riqueza e pobreza, essa atribuição defensiva é mais comum.

Algumas pessoas acreditam em um mundo justo por causa de sua experiência pessoal (funcionalismo individual), mas existem evidências que a crença no mundo justo não é apenas uma função da experiência pessoal, mas também do funcionalismo societal, isto é, fatores societais (Furnham, 2003). Em outras palavras, há condições situacionais (do ambiente) e disposicionais (da pessoa) que influenciam o processo de atribuição de BJW.

É consensual na literatura o fato de que a crença no mundo justo ajuda as pessoas a lidarem com perturbações ou eventos ameaçadores (estupro, pobreza, racismo), e que a experiência partilhada destes eventos leva as pessoas a desenvolverem uma visão consensual da realidade. A crença no mundo justo reduz ou evita sentimentos de culpa (Furnham, 2003). Durante os últimos anos, pesquisadores têm questionado se a crença no mundo justo é um construto unidimensional ou multidimensional. Esta inquietação teórica tem levado ao teste empírico, através de medidas objetivas do construto (Furnham, 2003; Mudrack, 2005). A propósito, a mensuração destas crenças é discutida a seguir. 


\section{Mensuração das crenças no mundo justo}

Com o objetivo de mensurar as crenças no mundo justo, têm-se desenvolvido medidas objetivas, tipo lápis e papel, que fazem uso de escalas tipo Likert. Tem-se como exemplo desta perspectiva de se conhecer o fenômeno, a Escala Global de Crenças do Mundo Justo (Global Beliefs in a Just World Scale; GJWS) de Lipkus (1991), que apresentou valor Alfa de Cronbach (precisão) de 0,83 no estudo original e evidências de validade concorrente. Dalbert e cols. (2001) por sua vez encontraram bons índices de consistência $(\alpha)$ variando de 0,65 a 0,72 para esta escala. Furnham (2003) por sua vez reporta valores de precisão variando de 0,79 a 0,83 para a GJWS. Além disso, recentemente esta medida foi testada juntamente com a Just World Scale (JWS) original e revisada por Hellman, Muilenburg-Trevino e Worley (2008) os quais verificaram que a escala de Lipkus apresentou o melhor coeficiente de precisão $(\alpha=0,81)$.

Esta medida foi desenvolvida com o objetivo de ser uma alternativa adequada para a Escala do Mundo Justo (JWS, Rubin \& Peplau, 1975), tipicamente utilizada para medir BJW (Edlund \& cols., 2007). A GJWS contém menos itens (apenas sete) do que a JWS, formada por 20 itens. A despeito da popularidade desta última, ela vem sendo criticada por apresentar propriedades psicométricas pouco desejáveis, como baixa consistência interna e uma estrutura fatorial inconsistente, levando ao desenvolvimento de outras medidas (Lucas, Alexander, Firestone \& Lebreton, 2007; Mudrack, 2005). Mudrack, por exemplo, explica que, de 1985 a 2002, os estudos sobre a JWS têm retido 1, 2, 4 e até 5 fatores. Desta forma, a GJWS pode ser considerada uma solução prática para mensuração das crenças no mundo justo, principalmente quando se busca testar um modelo complexo para explicação do construto, o qual, necessariamente, inclui outras medidas. Furnham (2003), neste sentido, tem verificado uma tendência nos pesquisadores de desenvolver questionários curtos para a mensuração das BJW; as quais, segundo ele, podem ser utilizadas em estudos correlacionais e experimentais. Nesta perspectiva, em realidade, torna-se muito menos provável que se coletem respostas dadas ao azar ou sem a ponderação adequada, devido à fadiga que pode gerar um questionário longo. Ademais, Gosling, Rentfrow e Swann (2003), explicam que, além da possibilidade de inclusão de outras medidas, estes questionários podem ser empregados favoravelmente em estudos que se pretendam comparar culturas. Isto pode ser eficaz para se entender como os indivíduos fazem julga- mentos no dia a dia, como percebem eventos vitimizadores ou, mais especificamente, como tomam decisões nos tribunais.

Com o fim de verificar a existência de uma escala publicada em versão portuguesa de acesso em periódicos de psicologia, realizou-se uma busca no Index Psi com a expressão "Escala de Crenças no Mundo Justo". De acordo com esta busca, não se conseguiu verificar nenhum resultado. Com o objetivo de cobrir outras revistas, foi realizada a mesma busca no Google Acadêmico, mas também não se evidenciou nenhum artigo. Esta área de pesquisa, entretanto, pode interessar tanto à psicologia social como à psicologia jurídica. Tendo isto em vista, o presente estudo visou validar por construto a GJWS em uma amostra de estudantes brasileiros através de uma abordagem fatorial confirmatória. Além disso, busca-se verificar a consistência interna desta medida e diferenças por variáveis sociodemográficas, com o fim de que esta medida possa ser uma alternativa para ser utilizada em estudos no contexto da psicologia brasileira

\section{MÉTODO}

\section{Participantes}

Foram escolhidos não-probabilisticamente, 254 alunos de uma universidade particular, no nordeste brasileiro, da cidade de Aracaju (Sergipe). Estes tinham média de idade de 21,83 anos $(\mathrm{dp}=6,34)$, sendo a maioria composta de mulheres $(63,8 \%)$, solteiros $(82,3 \%)$, de classe média $(61,8 \%)$ e do primeiro período (47,6\%). 49,6\% da amostra constituem-se por alunos do Curso de Direito e 44,9\% do Curso de Psicologia. Além disso, 90\% destes é de pessoas com idades compreendidas entre 16 e 30 anos e $43 \%$ dos participantes reportou nível médio de religiosidade.

\section{Instrumento}

Escala Global de Crenças do Mundo Justo. A GJWS construída por Lipkus (1991) é uma medida breve e unifatorial, composta de sete itens apenas, para aferir BJW, cujos itens são medidos numa escala do tipo Likert de 6 pontos, que variam de 1=Discordo Totalmente a $6=$ Concordo Totalmente. As crenças no mundo justo são medidas através de itens como "As pessoas ganham as recompensas e punições que merecem" ou "As pessoas conseguem o que merecem". Portanto, quanto mais próximo do ponto 6 mais endosso a crenças no mundo justo. A escala foi traduzida para o português com a participação de diversos 
pesquisadores. Ao final do questionário foram incluídas questões de caráter sociodemográfico, como sexo, idade, nível socioeconômico e curso para posteriores análises.

\section{Procedimento de coleta dos dados}

Inicialmente, buscou-se uma versão do questionário que fosse melhor compreendida pelos participantes. Para tanto, procurou-se, antes de tudo, validá-lo semanticamente, considerando o processo de tradução, as diferenças culturais e equivalência semântica. Portanto, antes da administração dos questionários na amostra total, procedeu-se a esta etapa.

\section{Validação semântica}

Segundo Pasquali (2003), para iniciar o processo de validação de escalas psicológicas, requere-se que estas sejam validadas semanticamente; tem a finalidade de verificar a compreensão e adequação dos itens, com uma amostra máxima de 10 pessoas que compõem a população de estrato mais baixo em relação àquela que o instrumento quer focar (neste caso foram considerados os alunos calouros, do primeiro semestre). Para tanto, a análise semântica foi realizada na mesma universidade, onde, posteriormente, o instrumento foi aplicado. A versão da escala, após suas devidas alterações, foi obtida nesta fase e considerada compreensível para ser administrada em estudantes universitários, considerando a clareza, objetividade e elegância na redação dos itens.

\section{Aplicação da versão resultante da validação semântica}

A versão resultante da validação semântica foi utilizada para a coleta dos dados na amostra total, composta por 250 estudantes universitários. Para tal, quatro estudantes de graduação de Psicologia, da Universidade Tiradentes, contataram os professores em suas devidas salas de aula, solicitando um tempo médio de 10 minutos para aplicação do instrumento com seus alunos. Para tanto, estes foram adequadamente informados que o objetivo da pesquisa tinha fins científicos. Aqueles dispostos a responder assinaram um termo de consentimento considerando as normas éticas para a condução de pesquisas com seres humanos. Os aplicadores, previamente treinados para o processo de coleta de dados, davam as instruções oralmente e garantiam total disponibilidade para qualquer dúvida quanto à forma de resposta, não de conteúdo, para evitar viés de resposta. Finalmente, após a aplicação, foram feitos agradecimentos de praxe pela participação voluntária da turma e a anuência do professor e da direção da escola.

\section{Análises dos dados}

Para a tabulação e as análises estatísticas dos dados, foi utilizado o SPSS (Statistical Package for the Social Science, versão 15). Utilizaram-se as estatísticas descritivas (medidas de tendência central e dispersão, distribuição de frequência e percentual) para caracterizar a amostra estudada. Para a validade fatorial, procedeu-se à análise de Componentes Principais (PC), com o objetivo de verificar a dimensionalidade do instrumento de medida. Além disso, os dados foram submetidos à análise da técnica Alfa de Cronbach. Em linhas gerais, trata-se de uma análise estatística dos dados de uma única aplicação de um teste a uma amostra representativa de sujeitos. Verifica a congruência que cada item do teste tem com o restante dos itens do mesmo teste. A fidedignidade do instrumento se refere à propriedade de ser consistente, medir com menor número de erros (Pasquali, 2003). É uma medida que varia de 0 a 1 , sendo o valor de 0,70 considerado o limite de aceitabilidade (Hair, Anderson, Tatham \& Black, 2006). Para realização de uma Análise Fatorial Confirmatória (CFA), optou-se pelo solfware AMOS 6 (Analysis of Moment Structures), considerando-se a matriz de covariâncias e adotando o método de estimação ML (Maximum Likelihood). Resumem-se, a seguir, alguns dos principais indicadores de qualidade de ajuste da análise do modelo:

- O Qui-quadrado $\left(\chi^{2}\right)$, que comprova a probabilidade do modelo se ajustar aos dados. Um valor do $\chi^{2}$ estatisticamente significativo indica discrepâncias entre os dados e o modelo teórico que está sendo testado. É influenciado pelo tamanho da amostra e assume a multinormalidade do conjunto de variáveis.

- O Índice de Qualidade do Ajuste (Goodness-ofFit Index, GFI) e o Índice de Qualidade do Ajuste Ajustado (Adjusted Goodness-of-Fit Index, AGFI), que é ponderado em função dos graus de liberdade do modelo, com respeito ao número de variáveis consideradas. É recomendado valores do GFI e AGFI superiores ou próximos a 0,95 e 0,90 , respectivamente (Garson, 2003; Hu \& Bentler, 1999; Tabachnick \& Fidel, 2001). Estas estatísticas não são influenciadas pelo tamanho da amostra dos participantes (Saris \& Stronkhorst, 1984). 
- O CFI (Comparative Fit Index) é um índice comparativo, adicional, de ajuste ao modelo, com valores mais próximos de 1 indicando melhor ajuste, com 0,90 sendo a referência para aceitar o modelo.

- Razão $\chi^{2} / g .1$ é considerada uma qualidade de ajuste subjetiva (Byrne, 1989). Um valor inferior a 5,00 pode ser interpretado como indicador da adequação do modelo teórico para descrever os dados.

- Raíz quadrada média do erro de aproximação (Root Mean Square Error of Approximation, RMSEA). Com relação aos valores de RMSEA, recomendam-se valores próximos a 0,06 e 0,08 , respectivamente (Hu \& Bentler, 1999) com seu intervalo de confiança de $90 \%$ (IC90\%), interpretam-se valores altos como indicação de um modelo não ajustado.

Foram realizadas também Análise de Variância (ANOVA) fatorial e análise de correlação de Pearson para verificar possíveis relações com as variáveis sociodemográficas consideradas em relação à pontuação total nas crenças no mundo justo.

\section{RESULTADOS}

\section{Análise dos componentes principais}

Antes de se proceder a análise dos componentes principais, buscou-se conhecer se a matriz de correlações era fatorável. De acordo com os índices de KMO $(0,75)$ e Teste de Esfericidade de Bartlett $\left(\chi^{2}(21)=\right.$ $301,614, \mathrm{p}<0,001)$ obtidos, pode-se comprovar a fatoriabilidade e proceder a análise PC. Desta análise, sem se fixar o método de rotação e o número de fatores a se extrair, emergiu uma estrutura bi-fatorial com dois valores próprios (eigenvalues) maiores que 1,0 (2,65 e $1,08)$ explicando $53,36 \%$ da variância total com cargas fatoriais variando de 0,39 a 0,64 . No entanto, por apresentar uma estrutura fatorial pouco clara, com itens saturando ao mesmo tempo nos dois fatores, e por se esperar unidimensionalidade da escala de acordo com o original, decidiu-se realizar uma análise paralela. O segundo valor próprio gerado pela análise paralela (Critério de Horn; ver Laros, 2005) com 1000 simulações foi de 1,14 , valor este que é superior ao segundo eigenvalue encontrado na PC $(1,08)$, indicando, assim, que a estrutura unidimensional deve ser optada. Tendo em vista a superioridade deste critério de retenção dos itens nos fatores em relação aos demais, realizou-se uma nova análise, fixando-se em um único fator.

TABELA

Estrutura Fatorial da GJWS

\begin{tabular}{lrr}
\hline ltens & Carga fatorial & $\mathrm{h}^{2}$ \\
\hline 03. As pessoas ganham as recompensas e punições que merecem. & 0,76 & 0,58 \\
06. Penso que as recompensas e punições são atribuídas justamente. & 0,66 & 0,43 \\
02. Quando uma pessoa se esforça, ela é reconhecida e recompensada. & 0,65 & 0,42 \\
05. As pessoas conseguem o que merecem. & 0,62 & 0,39 \\
01. As pessoas recebem o que elas têm o direito a ter. & 0,60 & 0,36 \\
04. As pessoas se encontram com o infortúnio que elas mesmas trazem. & 0,55 & 0,31 \\
07. Eu basicamente penso que o mundo é um lugar justo. & 0,39 & 0,15 \\
\hline Valor próprio (eigenvalue) & 2,65 & \\
Variância explicada (\%) & 37,89 & \\
Alfa de Cronbach & 0,71 & \\
\hline
\end{tabular}

Como se pode ver na Tabela 1 , desta análise surge um fator com eigenvalue $=2,65$, responsável por $37,90 \%$ da variância total e cargas fatoriais variando de 0,39 a 0,76. O gráfico scree plot (Critério de Cattel; ver Laros, 2005) também reforça esta solução unifatorial (ver Figura 1). 
Scree Plot

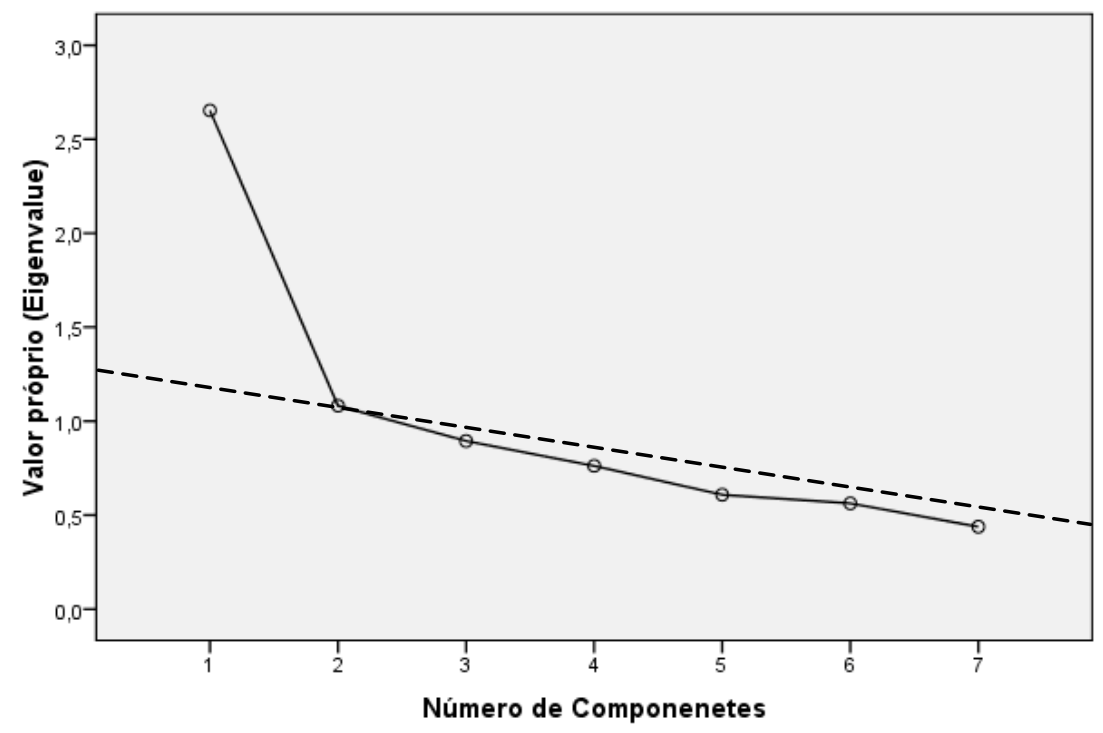

Figura 1. Distribuição gráfica do valor próprio da GJWS.

\section{Análise de consistência interna}

A consistência interna foi obtida mediante a técnica Alfa de Cronbach $(\alpha=0,71)$. O valor encontrado para a GJWS está dentro da margem de aceitabilidade. Assim, a precisão da escala pode ser considerada satisfatória.

\section{Análise fatorial confirmatória}

Com o objetivo de conseguir mais evidências para se corroborar a unidimensionalidade da escala, condu- ziu-se uma análise fatorial confirmatória (CFA) pelo método de estimação Maximum Likelihoood (ML). De acordo com a CFA, puderam-se observar os seguintes resultados: $\chi^{2} /$ g.l. $=3,37$; GFI $=0,95 ;$ AGFI $=0,90$; $\mathrm{CFI}=0,88$; RMSEA $=0,09(0,06-0,12)$. Todos os pesos fatoriais, lambdas $(\lambda)$, foram estatisticamente significativos e diferentes de zero $(p<0,001)$. Tal análise traz novas informações estatísticas que corroboram a unidimensionalidade da escala (Figura 2).

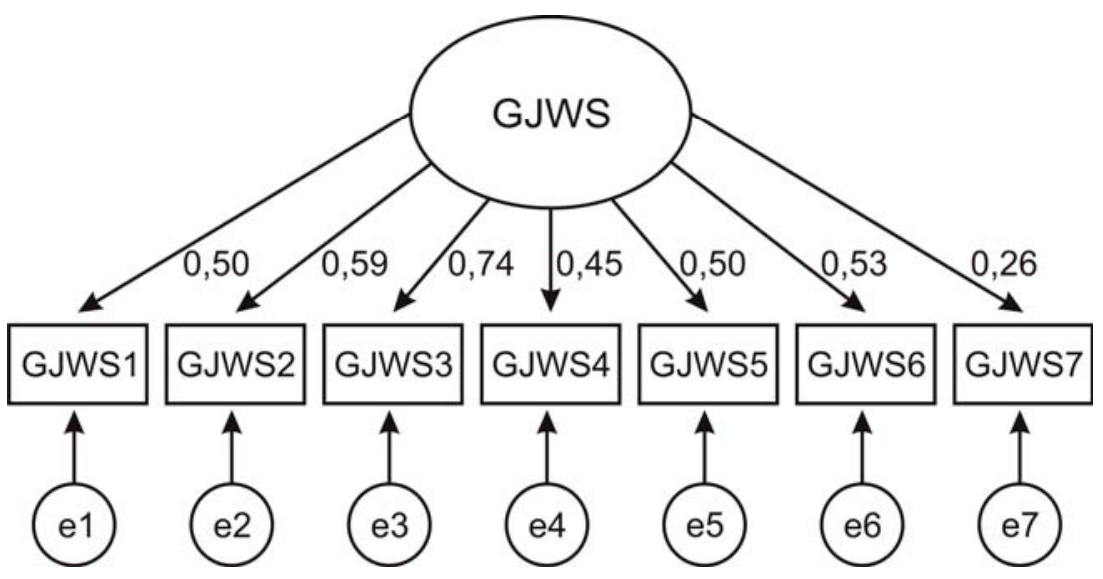

Figura 2. Análise fatorial confirmatória da GJWS. 


\section{Análises das variáveis sociodemográficas}

Com o objetivo de se conhecer mais sobre as crenças no mundo justo, realizou-se teste de comparação de média, considerando a pontuação total na GJWS ( $m=3,92, d p=0,78)$ que indica moderadas crenças no mundo justo. Considerou-se o nível socioeconômico, estado civil, período que se está cursando, sexo e curso como variáveis antecedentes para explicar as crenças no mundo justo. De acordo com a ANOVA fatorial, não se verificou interação entre as variáveis nem efeitos estatisticamente significativos destas variáveis nas crenças no mundo justo. Através da análise de correlação de Pearson, não se verificou nenhuma relação entre idade e BJW, nem entre nível de religiosidade e BJW. O nível de religiosidade se relacionou positivamente com a idade $(r=0,15, p=0,02)$, indicando que quanto mais idade, mais religiosos os participantes afirmaram ser.

\section{DISCUSSÃO}

O resultado mais palpável da verificação da validade fatorial e da precisão nesta pesquisa é contar com a versão da GJWS, prática (de fácil aplicação e manuseio) e de baixo custo (técnica de lápis e papel). Os resultados da análise PC indicam que mais de 50\% da variância total do construto é explicada, com consistência interna meritória - de acordo com a teoria clássica dos testes (precisão - Anastasi \& Urbina, 2000; Hair \& cols., 2006; Pasquali, 2003). A CFA aporta evidências adicionais para a unidimensionalidade da escala, que mostram a qualidade do ajuste do modelo que suporta a medida. De fato, os índices de adequação de ajuste correspondem à referência indicada na área (Byrne, 1989; Garson, 2003; Hu \& Bentler, 1999), ou estão muito próximos, especificamente o RMSEA (Hu \& Bentler, 1999).

A despeito de não se ter encontrado um excelente índice de precisão para a escala, como reportado em estudos prévios (Furnham, 2003; Hellman \& cols., 2008; Lipkus, 1991), a precisão da GJWS está acima do indicado pelo Conselho Federal de Psicologia, que tem recomendado um índice de consistência de pelo menos 0,60 (Couto, Vandenberghe, Hattum, \& Campos, 2006). Deve-se ponderar, ainda, que a GJWS não é dispendiosa, contando com um número reduzido de itens $(<10)$; sendo, pois, esperados menores coeficientes de precisão ( $\alpha$; Ledesma, Ibañez, \& Mora, 2002; Nunnally, 1991; Pasquali, 2003). Outra característica importante do estudo que influencia a precisão da escala é o tamanho da amostra e a variabilidade da mesma (Nunnally, 1991; Pasquali, 2003). Ou seja, se acrescentados novos itens nesta medida, e testando-a numa amostra maior e mais variável, certamente conseguir-se-ia um índice Alfa de Cronbach melhor. Embora o utilizado nesta pesquisa seja um quantitativo de participantes adequado para as análises efetuadas (Hoyle, 1995).

Uma vez que a concepção BJW como um construto unidimensional medido pela GJWS é reforçada, estes achados podem jogar luz no debate sobre a estrutura fatorial, a dimensionalidade destas crenças (Dalbert \& cols., 2001; Furnham, 2003; Mudrack, 2005). A GJWS pode ser considerada uma ferramenta válida, prática e precisa para se conhecer a extensão das crenças no mundo justo de estudantes universitários, seja em estudos correlacionais ou experimentais, independente das variáveis sociodemográficas consideradas. De fato, uma escala curta (menos de dez itens) favorece a unidimensionalidade do construto em pauta. Entretanto, além da perspectiva dos estudos na área considerarem esta estratégia (Furnhan, 2003), existem vantagens reportadas na literatura especializada (Gosling \& cols., 2003). De fato, com uma medida breve de BJW seria mais fácil incluir outras variáveis em um modelo de equações estruturais (Byrne, 2001) ou de regressão multinível (Laros \& Marciano, 2008; Maas \& Hox, 2004). Por outro lado, como comentam os autores (Gosling \& cols., 2003), não é o caso de dispensar o uso das escalas convencionais, mas poder contar com escalas que meçam o construto para serem utilizadas em situações específicas, que não se demande muito tempo. Como se pôde observar, não foram verificadas diferenças nas crenças do mundo justo em razão do sexo dos participantes, idade, curso, nível socioeconômico, religiosidade, estado civil ou o período que se está cursando.

Quanto ao sexo, numa revisão de vários estudos (através de meta-análise), verificou-se que os homens (de menor status socioeconômico) tendem a crer mais no mundo justo do que as mulheres, mesmo sendo uma diferença sutil (Furnhan, 2003). Porém, o próprio autor indica que também não se têm encontrado diferenças quanto ao sexo nestas crenças e uma modesta associação com a religiosidade, e apresenta estudos que mostram que os mais religiosos endossam mais crenças no mundo justo (ver, por exemplo, Dalbert \& cols., 2001). No presente estudo, não se confirmaram diferenças nas crenças no mundo justo em função da religiosidade nem do sexo dos participantes, assim como não se puderam verificar diferenças quanto à renda percebida dos participantes. Furnhan (2003) realmente destaca que "o maior suporte para as crenças no mundo justo vem dos homens de menor status 
sócio-econômico" (p. 810). Porém, aqui, esta evidência não é suportada empiricamente. Quanto à idade, Dalbert e colaboradores (2001) também verificaram na sua pesquisa que os resultados para a GJWS independeu da idade dos participantes.

É importante que estes achados sejam replicados em outras culturas para que se possa (a) verificar se os resultados se repetem, (b) se outras versões da escala (em espanhol, p. ex.) podem ser usadas em diferentes contextos ou (c) para checar suas relações com dimensões culturais (Furnham, 2003). Certamente, aponta-se como a maior limitação da presente pesquisa a composição da amostra e a técnica de amostragem. Por razões inerentes à acessibilidade e calendário de aulas, contou-se apenas com a participação dos alunos da rede privada de ensino, que foram escolhidos de modo não-aleatório. Estes alunos foram todos do ensino superior, com todas as características sociodemográficas conforme apresentadas. Todos estes aspectos devem ser tidos em consideração na interpretação desses resultados. Adite-se a isso a própria natureza dos dados, oriunda de um estudo delimitado temporalmente, do tipo cross-sectional (ver Dalbert \& cols., 2001). Estas peculiaridades limitam o poder de generalização dos dados, mas considera-se este como um estudo muito mais voltado para teste psicométrico da GJWS.

\section{CONCLUSÃO}

Em suma, foram reunidas evidências de validade fatorial, consistência interna e relação com variáveis sociodemográficas para a GJWS. Outras pesquisas deveriam considerar os limites e possibilidades ora apontados, incluindo pesquisas com alunos da rede pública, diferentes escolaridades, níveis de religiosidade e/ou afiliações religiosas e preferencialmente de diferentes estados e regiões. É importante ademais avaliar a sua consistência temporal através da técnica de teste-reteste (Anastasi \& Urbina, 2000). Sem dúvidas, estes e outros empreendimentos contribuirão para um maior conhecimento das variáveis relacionadas às BJW, contribuindo, assim, para a teoria e mensuração destas crenças.

\section{REFERÊNCIAS}

Anastasi, A., \& Urbina, S. (2000). Testagem psicológica (M. A. V. Veronese, Trad.). Porto Alegre: ArtMed.

Aronson, E., Wilson, T. D., \& Akert, R. M. (2002). Psicologia social (R. Jungmann, Trad.). Rio de Janeiro: LTC.

Bègue, L. (2002). Beliefs in justice and faith in people: Just world, religiosity and interpersonal trust. Personality and Individual Differences, 32, 375-382.
Bègue, L., \& Muller, D. (2006). Belief in a just world as moderator of hostile attributional bias. British Journal of Social Psychology, 46, 117-126.

Byrne, B. M. (1989). A primer of LISREL: Basic applications and programming for confirmatory factor analytic models. New York: Springer-Verlag.

Byrne, B. M. (2001). Strutural equation modeling with Amos: Basic concepts, applications, and programming. Londres: Lawrence Erlbaum.

Correia, I., \& Vala, J. (2003). Crença no mundo justo e vitimização secundária: O papel moderador da inocência da vítima e da persistência do sofrimento. Análise Psicológica, 21(3), 341-352.

Couto, G., Vandenberghe, M. A., Hattum, C. F. M. V., \& Campos, H. R. (2006). Propriedades psicométricas do checklist de relações interpessoais. Psicologia Argumento, 24, 15-28.

Dalbert, C., Lipkus, I.M., Sallay, H., \& Goch, I. (2001). A just and an unjust world: Structure and validity of different world beliefs. Personality and Individual Differences, 30, 561-577.

Edlund, J. E., Sagarin, B. J., \& Johnson, B. S. (2007). Reciprocity and the belief in a just world. Personality and Individual Differences, 43, 589-596.

Furnham, A. (1995). The just world, charitable giving and attitudes to disability. Personality and Individual Differences, 19(4), 577-583.

Furnham, A. (2003). Belief in a just world: Research progress over the past decade. Personality and Individual Differences, 34, 795-817.

Furnham, A., \& Procter, E. (1989). Belief in a just world: Review and critique of the individual difference literature. British Journal of Social Psychology, 28, 365-384.

Garson, G. D. (2003). PA 765 Statnotes: An online textbook. Retirado em 17 de maio de 2005, de http://www2.chass.ncsu. edu/garson/pa765/statnote.htm

Gosling, S. D., Rentfrow, P. J., \& Swann Jr., W. B. (2003). A very brief measure of the big-five personality domains. Journal of Research in Personality, 37, 504-528.

Hair, J. F. Jr., Anderson, R. E., Tatham, R. L., \& Black, W. C. (2006). Análise multivariada (A. S. Sant'Anna, Trad.). Porto Alegre: Bookman.

Hellman, C. M., Muilenburg-Trevino, E. M., \& Worley, J. A. (2008). The belief in a just world: An examination of reliability estimates across three measures. Journal of Personality Assessment, 90(4), 399-401.

Hoyle, R. (1995). Structural equation modeling: Concepts, issues and applications. Thousand Oaks, CA: SAGE Publications.

Hu, L. T., \& Bentler, P. M. (1999). Cutoff criteria for fit indexes in covariance structure analysis: Conventional criteria versus new alternatives. Structural Equation Modeling, 6, 1-55.

Laros, J. A. (2005). O uso da análise fatorial: Algumas diretrizes para pesquisadores. Em L. Pasquali (Org.), Análise fatorial para pesquisadores (pp. 163-184). Brasília: LabPAM.

Laros, J. A., \& Marciano, J. L. P.(2008). Análise multinível aplicada a dados do NELS:88. Estudos em Avaliação Educacional, 19(40), 263-278. 
Ledesma, R., Ibañez, G. M., \& Mora, P. V. (2002). Análisis de consistencia interna mediante Alfa de Cronbach: Un programa basado en gráficos dinámicos. Psico-USF, 7, 143-152.

Lerner, M. J. (1980). The belief in a just world: A fundamental decision. New York: Plenum.

Lerner, M. J. (1998). The two forms of belief in a just world: Some thoughts on why and how people care about justice. Em L. Montada \& M. J. Lerner (Orgs.), Responses to victimizations and belief in a just world (pp. 247-270). New York: Plenum Press.

Lerner, M. J. (2003). The justice motive: Where social psychologist found it, how they lost it, and why they may not find it again. Personality and Social Psychology Review, 7, 388-399.

Lerner, M. J., \& Miller, D. T. (1978). Just world research and the attribution process: Looking back and ahead. Psychological Bulletin, 85, 1030-1051.

Lipkus, I. (1991). The construction and preliminary validation of a global belief in a just world scale and the exploratory analysis of the multidimensional belief in a just world scale. Personality and Individual Differences, 12, 1171-1178.

Lucas, T., Alexander, S., Firestone, I., \& LeBreton, J. M. (2007). Development and initial validation of a procedural and distributive just world measure. Personality and Individual Differences, 43, 71-82.

Maas, C. J. M., \& Hox, J. J. (2004). Robustness issues in multilevel regression analysis. Statistica Neerlandica, 58(2), 127137.

Montada, L. (2003). Justice, equity, and fairness in human relations. Em T. Milton \& M. J. Lerner (Orgs.), Personality and social psychology: Vol. 5 (pp. 537-568). New Jersey: John Wiley \& Sons.
Mudrack, P. E. (2005). An outcomes-based approach to just world beliefs. Personality and Individual Differences, 38, 817-830.

Nunnally, J. C. (1991). Teoría psicométrica. México: Trillas.

Otto, K., Boos, A., Dalbert, C., Schöps, D., \& Hoyer, J. (2006). Posttraumatic symptoms, depression, and anxiety of flood victims: The impact of the belief in a just world. Personality and Individual Differences, 40, 1075-1084.

Pasquali, L. (2003). Psicometria: Teoria dos testes na psicologia e na educação. Petrópolis: Vozes.

Rodrigues, A., Assmar, E. M. L., \& Jablonski, B. (2000). Psicologia social. Petrópolis: Vozes.

Rubin, Z., \& Peplau, L. A. (1975). Who believes in a just world? Journal of Social Issues, 31, 65-90.

Saris, W., \& Stronkhorst, H. (1984). Causal modeling in nonexperimental research. Amsterdam: Sociometric Research Foundation.

Strelan, P. (2007). The prosocial, adaptive qualities of just world beliefs: Implications for the relationship between justice and forgiveness. Personality and Individual Differences, 43, 881890.

Tabachnick, B. G., \& Fidell, L. S. (2001). Using multivariate statistics. Boston, MA: Allyn \& Bacon.

Wolfradt, U., \& Dalbert, C. (2003). Personality, values and belief in a just world. Personality and Individual Differences, 35(8), 1911-1918.

Recebido: $24 / 10 / 2008$

Última revisão: 05/03/2010

Aceite final: $12 / 03 / 2010$

\section{Sobre os autores:}

Valdiney V. Gouveia: Doutor em Psicologia Social pela Universidade Complutense de Madrid, Professor Adjunto da Universidade Federal da Paraíba.

Endereço para correspondência: Universidade Federal da Paraíba. Doutorado Integrado em Psicologia Social. CCHLA - Departamento de Psicologia. 58051-900 João Pessoa - PB. Endereço eletrônico: vvgouveia@pesquisador.cnpq.br.

Carlos Eduardo Pimentel: Doutorando em Psicologia Social, do Trabalho e das Organizações na Universidade de Brasília. Bolsita do CNPq.

Endereço para correspondência: Programa de Pós-graduação em Psicologia Social, do Trabalho e das Organizações - Instituto de Psicologia - Universidade de Brasília - Campus Universitário Darcy Ribeiro - Asa Norte - Caixa Postal 4500 - 70919-970 Brasília DF. Endereço eletrônico: carlospimentel@unb.br ou carlosepimentel@bol.com.br.

Jorge Artur P. de M. Coelho: Doutor em Psicologia Social pela Universidade Federal da Paraíba, Professor Adjunto da Universidade Federal de Alagoas.

Endereço para correspondência: Universidade Federal de Alagoas, Instituto de Ciências Humanas, Comunicação e Artes, Coordenação de Psicologia, Campus A. C. Simões, BR 104 Norte, Km 14, Tabuleiro do Martins, 57072-970 Maceió - AL. Endereço eletrônico: jorgearturpmc@gmail.com.

Viviane Andrade Prado Maynart: Aluna do Curso de Graduação em Psicologia na Universidade Tiradentes. Endereço para correspondência: Universidade Tiradentes. Curso de Psicologia. Av. Mutilo Dantas, 300 - Farolândia - $49032-490$ Aracaju - SE. Endereço eletrônico: viviandradeprado@hotmail.com.

Tamara dos Santos Mendonça: Aluna do Curso de Graduação em Psicologia na Universidade Tiradentes. Endereço para correspondência: Universidade Tiradentes. Curso de Psicologia. Av. Mutilo Dantas, 300 - Farolândia - 49032-490 Aracaju - SE. Endereço eletrônico: tamara_psic@hotmail.com. 